\title{
WEIGHTED CAPACITY AND THE CHOQUET INTEGRAL
}

\author{
DAVID R. ADAMS
}

(Communicated by William C. Waterhouse)

\begin{abstract}
The capacity set function that is naturally associated with a linear second-order elliptic partial differential operator in divergence form is related to the concept of the Choquet integral of a weight function with respect to Newtonian capacity. The weight function comes from the coefficients of the differential operator. This idea is reminiscent of the Radon-Nikodym Theorem, but now for capacities instead of measures.
\end{abstract}

1. Introduction. Weighted capacity is to a linear second-order elliptic partial differential equation in divergence form as Newtonian capacity is to the Laplace equation. In the more general setting, the weighted capacity is built out of the coefficients of the differential operator. In this note, we will represent these weighted capacities by the symbol $C^{w}$, where $w$ is the weight-a nonnegative (Borel) measurable function on Euclidean $n$-space $\mathbf{R}^{n}$. The relationship between the weight and the differential operator is through the ellipticity condition. For the operator $\mathbf{L} u=-\left(a_{i j}(x) u_{x_{i}}\right)_{x_{j}}$, with the summation convention, the condition is

$$
Q^{-1}|\xi|^{2} w(x) \leq a_{i j}(x) \xi_{i} \xi_{j} \leq Q|\xi|^{2} w(x)
$$

for all $x$ and $\xi$ in $\mathbb{R}^{n}$. Here $Q$ is some positive constant. If $w$ is allowed to be zero or plus infinity, then the operator $\mathbf{L}$ is termed degenerate. Clearly, if the degeneracy is bad enough, then the behavior of solutions to $\mathbf{L} u=0$ can differ drastically from those to $\Delta u=0$, the Laplace equation. However, when $w$ is bounded above and below by positive constants, then the local continuity properties of $\mathbf{L} u=0$ solutions closely resemble those for $\Delta u=0$ solutions. This is basically the theme of the papers [LSW and S]. If the weights have enough regularity, though short of being bounded above and below, then a theory of degenerate equations can be constructed that closely resembles the nondegenerate one. This can be found in [MS, EP, FKS, FJK]. In either case, degenerate or nondegenerate, a measure of the deviation from continuity for solutions is the concept of capacity, weighted or unweighted. This is of interest near points of singular sets or in the neighborhood of boundary points for Dirichlet regions. Our interest here, however, is not to describe these theories, but to examine these weighted capacities with the idea of comparing them with the Choquet integral of the weight with respect to Newtonian capacity (see the definition below). This idea is reminiscent of the Radon-Nikodym Theorem, but now for capacities and capacitary (Choquet) integrals.

Received by the editors January 22, 1987. Presented at the Workshop on Weighted Norm Inequalities and Applications, Université de Montréal, October 29-31, 1987.

1980 Mathematics Subject Classification (1985 Revision). Primary 35J15, 31C15.

This research was supported (in part) with funds from the National Science Foundation (Grant No. RII-8610671) and the Commonwealth of Kentucky through the Kentucky EPSCoR Program. 
2. Preliminaries. If $w \geq 0$, we define the weighted capacity $C^{w}(K)$ of a compact subset $K \subset \mathbb{R}^{n}$ by

$$
C^{w}(K)=\inf \int|\nabla \phi(x)|^{2} w(x) d x
$$

where the infimum is over all $\phi \in C_{0}^{\infty}\left(\mathbb{R}^{n}\right)$ such that $\phi \geq 1$ on $K$. Here $\nabla \phi$ is the gradient of $\phi$. When $w \equiv 1$, we will write $C(K)$. These capacities can be extended to all sets by: for $G$ open

$$
C^{w}(G)=\sup _{K \subset G} C^{w}(K)
$$

and for $E$ arbitrary

$$
C^{w}(E)^{*}=\inf _{E \subset G} C^{w}(G) .
$$

By the general results of Choquet $[\mathbf{C}]_{2}, C^{w}(E)^{*}=C^{w}(E)$ for all analytic sets $E$.

Let $\dot{W}^{1,2}(w)$ denote the closure of $C_{0}^{\infty}\left(\mathbb{R}^{n}\right)$ with respect to $\left(\int|\nabla \phi|^{2} w d x\right)^{1 / 2}$. When $w \equiv 1$, write $\dot{W}^{1,2}$. The (capacity) extremal for $C^{w}(K), K$ compact, is the unique (when it exists) $\phi \in \dot{W}^{1,2}(w)$ for which equality occurs in (1).

A weight $w$ is said to be an $A_{p}$ weight, or $w \in A_{p}$ for short, if for all balls $B \subset \mathbb{R}^{n}$

$$
\sup _{B}\left(f_{B} w d x\right)\left(f_{B} w^{-1 /(p-1)} d x\right)^{p-1}<\infty, \quad 1 \leq p<\infty
$$

(the barred integral means "average"). $A_{\infty}=\bigcup_{p<\infty} A_{p}$. See [CF]. When $w \in A_{2}$, it is easy (using the results of $[\mathbf{C F}]$ ) to see that

$$
C^{w}(K) \sim \inf \int f(x)^{2} w(x) d x
$$

where the infimum is over all measurable $f(x) \geq 0$ on $\mathbb{R}^{n}$ such that the Riesz potential $I_{1} * f(x)=\int|x-y|^{1-n} f(y) d y \geq 1$ on $K$. Here the symbol $\sim$ is read "is comparable to" and means that the ratio of the two quantities is bounded above and below by two positive constants-independent of $K$. Weighted capacities given by (2) have been studied recently in $\left[\mathbf{A}_{5}\right]$.

An important property of the capacities $C^{w}$ is strong subadditivity:

$$
C^{w}\left(K_{1} \cup K_{2}\right)+C^{w}\left(K_{1} \cap K_{2}\right) \leq C^{w}\left(K_{1}\right)+C^{w}\left(K_{2}\right)
$$

for any two compact sets $K_{1}$ and $K_{2}$. This is stronger than the usual subadditivity condition where the term involving $K_{1} \cap K_{2}$ in (3) is omitted. The reason this condition is important is because it is intimately related to the idea of the Choquet integral of a function $\phi \geq 0$ with respect to a general capacity, say $\Gamma$. By this we shall mean

$$
\int \phi d \Gamma \equiv \int_{0}^{\infty} \Gamma([\phi>t]) d t
$$

In $\left[\mathbf{C}_{1}\right]$, it is shown that such an integral is sublinear in $\phi$ if and only if $\Gamma$ is strongly subadditive; see also [An]. Thus, it follows in the standard way that the expression $\left(\int|\phi|^{p} d \Gamma\right)^{1 / p}, 1 \leq p<\infty$, is a norm when $\Gamma$ is strongly subadditive. Furthermore, the analogue of the Hölder inequality remains valid in this setting. And this all suggests that an obvious class of functions to consider is the class $L^{p}(\Gamma)$, which is the closure of the continuous functions on $\mathbb{R}^{n}$ with compact support with respect 
to the above norm. These spaces have been studied by the author and others, especially by $\mathrm{K}$. Hansson. See $\left[\mathbf{A}_{4}, \mathbf{H a}\right]$. In fact, Hansson characterizes these spaces as those $\Gamma$-quasi-continuous $\phi$ with finite norm. $\phi$ is $\Gamma$-quasi-continuous on $\mathbb{R}^{n}$ if, for every $\varepsilon>0$, there is an open set $G$ such that $\Gamma(G)<\varepsilon$, and $\phi$ restricted to the complement of $G$ is continuous there.

Below we will also have need of the so-called capacitary strong-type inequality (CSI) and some of its consequences. For this, see $\left[\mathbf{A}_{4}\right.$ or $\left.\mathbf{A}_{2}\right]$. There is a constant $Q$ such that for all $f \geq 0$

$$
\int\left(I_{1} * f\right)^{2} d C \leq Q \cdot \int f(x)^{2} d x
$$

From this it immediately follows that for $\psi \geq 0$

$$
\int \psi^{2} d C \leq Q^{\prime} \cdot C(\psi)
$$

where

$$
C(\psi)=\inf \int|\nabla \phi|^{2} d x
$$

with the infimum over all $\phi=I_{1} * f, f \geq 0$, such that $\phi \geq \psi, C$-a.e. It is also of interest to notice that (5) can be reversed, i.e. there is a constant $Q^{\prime \prime}$ such that

$$
C(\psi) \leq Q^{\prime \prime} \int \psi^{2} d C
$$

and thus, in particular, it follows that $\int \psi^{2} d C<\infty$ iff there is such a $\phi \geq \psi, C$-a.e. as above.

3. Statement of results. The question of interest for us in this note is the relationship between $C^{w}(K)$ and $\int_{K} w d C$. To motivate the main result, we first show

THEOREM 1. For all $w \in L^{2}(C)^{+}$,

$$
\lim _{r \rightarrow 0} f_{B(x, r)} w d C=w(x)
$$

for $C$-a.e. $x$. Here $B(x, r)=$ ball centered at $x$ of radius $r$.

This result can be found in more generality in $\left[\mathbf{A}_{3}\right]$. Again, the barred integral denotes "average" - now with respect to $C$. Also we will show

THEOREM 2. For all $w \in A_{2} \cap L^{2}(C)^{+}$,

$$
\lim _{r \rightarrow 0} \frac{C^{w}(B(x, r))}{C(B(x, r))}=w(x)
$$

for $C$-a.e. $x$.

Thus because of these results, we are tempted to ask for a Radon-Nikodym-type theorem here, i.e. when is it true that $C^{w}(K)=\int_{K} w d C$ ? It is obvious when $w$ is 
constant. We will show

THEOREM 3. (a) If $w$ is superharmonic on $\mathbb{P}^{n}$, then

$$
C^{w}(K) \leq \int_{K} w d C
$$

for all compact sets $K$.

(b) If, in addition, $w$ is of the form $I_{2} * v(x)=\int|x-y|^{2-n} v(y) d y$, the Newtonian potential of $v \geq 0$, where $v \in A_{\infty}$, then there is a constant $Q$ such that

$$
\int_{K} w d C \leq Q \cdot C^{w}(K)
$$

for all compact sets $K$.

Notice that if $w(x)=|x|^{-\beta}$, with $0<\beta<n-2$, then $w$ is superharmonic and we can take $v(x)=$ const $|x|^{-\beta-2}$, which satisfies the conditions set in the theorem. Actually, the results of $\left[\mathbf{A}_{5}\right]$ give that (9) and (10) are valid for $w(x)=|x|^{-\beta}$ whenever $-n<\beta<n-2$. This is a consequence of the hypothesis

$$
\sup _{A_{k}} w \leq Q \inf _{A_{K}} w, \quad K=1,2, \ldots
$$

made in $\left[\mathbf{A}_{5}\right]$ where $A_{K}$ is the annulus $\left\{x: 2^{-K-1}<|x| \leq 2^{-K}\right\}$.

For the weight $W_{\xi}(x)=|x-\xi|^{2-n}$ for $|x-\xi|<1$, and $W_{\xi}(x)=1$ for $|x-\xi| \geq 1$, the Choquet integral $\int_{K} W_{\xi} d C$ is just the Wiener test integral

$$
\int_{0}^{\infty} r^{2-n} \cdot C(K \cap B(\xi, r)) r^{-1} d r
$$

$K$ compact. The Kellogg property implies that this integral is infinite for $C$-a.e. $\xi \in K$. However, $C^{W_{\xi}}(K) \leq C^{W_{\xi}}(B(\xi, R))$ for some $R<\infty$, hence (10) must fail for $\beta=n-2$.

4. Proof of Theorem 1. This result will follow by the standard arguments once we show that the corresponding maximal function satisfies the appropriate weak-type condition. Our maximal function is

$$
\mathbf{M} \phi(x)=\sup _{r>0}\left[f_{B(x, r)} \phi^{2} d C\right]^{1 / 2}
$$

and we show

LEMMA 1. If $\phi \in L^{2}(C)^{+}$, then there is a constant $Q$ such that

$$
C([\mathbf{M} \phi>t]) \leq Q / t^{2} \int \phi^{2} d C .
$$

PROOF. By what was mentioned earlier, the finiteness of the right side of (11) implies that there is a funciton $f \geq 0$ s.t. $I_{1} * f \geq \phi, C$-a.e. Setting $f_{1}$ equal to $f$ on $B(x, 2 r)$ and zero otherwise, $f=f_{1}+f_{2}$, we can write

$$
\int_{B(x, r)}\left(I_{1} * f_{1}\right)^{2} d C \leq Q\left\|f_{1}\right\|_{2}^{2}
$$


and

$$
\int_{B(x, r)}\left(I_{1} * f_{2}\right)^{2} d C \leq 2^{2 n-2}\left(I_{1} * f(x)\right)^{2} \cdot C(B(x, r)) .
$$

Thus,

$$
\mathbf{M} \phi(x) \leq Q^{\prime}\left(M_{2} f^{2}(x)\right)^{1 / 2}+Q^{\prime \prime} I_{1} * f(x),
$$

where $M_{2} g(x)$ is the fractional maximal function of order 2 of $g$, i.e.

$$
\sup _{r>0} r^{2} f_{B(x, r)} g d y \text {. }
$$

Now we need

$$
C(K) \leq Q \cdot H^{n-2}(K) \text { for all compact } K
$$

and

where

$$
H^{n-2}\left(\left[M_{2} g>t\right]\right) \leq \frac{Q}{t} \int g d y \text { for all } t>0
$$

$$
H^{n-2}(E)=\inf \sum_{j} r_{j}^{n-2}
$$

with the infimum over all countable coverings of $E$ by balls $B_{j}$ (of radius $r_{j}$ ). For these facts, see $\left[\mathbf{A}_{4}\right]$ or $[\mathbf{M}]$ and $[\mathbf{B Z}]$. Thus, it follows that

$$
C([\mathbf{M} \phi(x)>t]) \leq \frac{Q}{t^{2}} \int f(y)^{2} d y,
$$

and hence with (6) the desired result is established.

Theorem 1 now follows by the usual methods in differentiation theory: the maximal function is of weak-type and the continuous functions are dense. In particular, we can replace $\phi$ by $|\phi-\phi(x)|$ and assert that

$$
\lim _{r \rightarrow 0} f_{B(x, r)}|\phi-\phi(x)|^{2} d C=0 .
$$

Thus, the result of the theorem is obtained from this, Hölder's inequality, and the sublinearity of the Choquet integral.

5. Proof of Theorem 2. First of all, it should be noted that from $\left[\mathbf{A}_{5}\right]$

$$
C^{w}(B(x, r)) \sim\left(\int_{r}^{\infty} \frac{t^{2}}{w(B(x, t))} \frac{d t}{t}\right)^{-1} .
$$

Thus, it is easy to see that the ratio $C^{w}(B(x, r)) / C(B(x, r))$ is comparable to $w(x)$, for $C$-a.e. $x$, when $r$ is sufficiently small.

The technique for establishing (8) is akin to the "blow-up" method in differential equations-we rescale and then pass to the limit. Thus, let $\phi_{r}$ be the extremal for $C^{\bar{w}}\left(B\left(x_{0}, r\right)\right)$. Here $\bar{w}$ is $w$ when $1 / N \leq w \leq N$ and $N$ or $1 / N$ corresponding as to when either $w>N$ or $w<1 / N$. This extremal is a weak solution to $\operatorname{div}(\bar{w} \nabla \phi)=0$ in the complement of $B\left(x_{0}, r\right)$. Furthermore, $0 \leq \phi_{r} \leq 1$ and $\phi_{r}=1$, $C$-a.e. on $B\left(x_{0}, r\right)$. Now set $x-x_{0}=r y$. Then with $\psi_{r}(y)=\phi_{r}\left(x_{0}+r y\right)$, the ratio becomes

$$
\frac{C^{\bar{w}}\left(B\left(x_{0}, r\right)\right)}{C\left(B\left(x_{0}, r\right)\right)}=\frac{\int\left|\nabla \psi_{r}(y)\right|^{2} \bar{w}\left(x_{0}+r y\right) d y}{C(B(0,1))} .
$$


Thus there is a subsequence of $\psi_{r}$ that converges weakly in $\dot{W}^{1,2}$ to some $\psi_{0} \in \dot{W}^{1,2}$ as $r \rightarrow 0$, i.e. the gradients converge weakly in $L^{2}$. We will show now that $\psi_{0}$ is in fact the extremal for $C(B(0,1))$. Notice first of all that $\psi_{0}=1, C$-a.e. on $B(0,1)$; cf. Theorem 5 of $[\mathbf{M}]$. Also by the change of variables given above, we can write

$$
\int \nabla \psi_{r}(y) \nabla \phi(y) \bar{w}\left(x_{0}+r y\right) d y=0
$$

for all $\phi \in C_{0}^{\infty}\left(\mathbb{R}^{n} \backslash B\left(x_{0}, r\right)\right)$. But by the Banach-Saks Theorem, we can pass to the limit and conclude that for $C$-a.e. $x_{0}$

$$
\int \nabla \psi_{0}(y) \nabla \phi(y) \bar{w}\left(x_{0}\right) d y=0
$$

Hence, we get $\Delta \psi_{0}=0$ away from $B(0,1)$ and the desired result follows. Notice that we have used the fact that

$$
\lim _{r \rightarrow 0} \int_{|y|<1}|w(x+r y)-w(x)|^{2} d y=0
$$

for $C$-a.e. $x$. This follows from the fact that the Hardy-Littlewood maximal function $M_{0}$ is of strong type on $L^{2}(C)$, i.e.

$$
\int\left(M_{0} \phi\right)^{2} d C \leq Q \int \phi^{2} d C
$$

and hence the averages $f_{B(x, r)} \phi d y$ converge $C$-a.e. $x$ to $\phi(x)$. Estimate (12) is an immediate consequence of (5) and (6), and the $L^{2}$-boundedness of $M_{0}$; cf. [ $\left.\mathbf{A}_{1}\right]$.

Finally, we argue by lower-semicontinuity that for $C$-a.e. $x_{0}$

$$
\begin{aligned}
\bar{w}\left(x_{0}\right) & =\frac{\int\left|\nabla \psi_{0}\right|^{2} \bar{w}\left(x_{0}\right) d x}{C(B(0,1))} \\
& \leq \lim _{r \rightarrow 0} \frac{\int\left|\nabla \psi_{r}(y)\right|^{2} \bar{w}\left(x_{0}+r y\right) d y}{C(B(0,1))} \\
& \leq \lim _{r \rightarrow 0} \frac{C^{\bar{w}}\left(B\left(x_{0}, r\right)\right)}{C\left(B\left(x_{0}, r\right)\right)} \\
& \leq \lim _{r \rightarrow 0} \frac{C^{w}\left(B\left(x_{0}, r\right)\right)}{C\left(B\left(x_{0}, r\right)\right)} .
\end{aligned}
$$

Now let $N \rightarrow \infty$.

To get an upper estimate, choose the test function $\phi(x)=h\left(r^{-1}\left|x-x_{0}\right|\right)$, where $h(x)=|x|^{2-n}$ for $|x|>1$ and $=1$ for $|x| \leq 1$. Then

$$
\frac{C^{w}\left(B\left(x_{0}, r\right)\right)}{C\left(B\left(x_{0}, r\right)\right)} \leq(n-2) r^{n-2} \int_{r}^{\infty} t^{2-n} W(t) \frac{d t}{t},
$$

where $W(t)=f_{S_{n-1}} w\left(x_{0}+t \xi\right) d \xi$. Since the left side of (13) is bounded away from zero for small $r$ and $C$-a.e. $x_{0}$, we have

$$
\varliminf_{r \rightarrow 0} \frac{C^{w}\left(B\left(x_{0}, r\right)\right)}{C\left(B\left(x_{0}, r\right)\right)} \leq \lim _{r \rightarrow 0} W(r)=w\left(x_{0}\right)
$$

for $C$-a.e. $x_{0}$. The last statement follows since the spherical maximal function

$$
w^{*}\left(x_{0}\right)=\sup _{t>0} f_{S^{n-1}} w\left(x_{0}+t \xi\right) d \xi
$$


also satisfies a strong-type inequality like (12) due to the results of [St] and estimates (5) and (6); $n \geq 3$.

6. Proof of Theorem 3. To prove part (a), we first choose an open set $G \supset K$ and let $\phi_{G}$ be the extremal for $C(G)$. Then upon integrating by parts and recognizing that $-\Delta \phi_{G}=\mu_{G}$, a Borel measure supported by $\bar{G}$, we have

$$
\int\left|\nabla \phi_{G}\right|^{2} w d x=\int \phi_{G} w d \mu_{G}-\frac{1}{2} \int \phi_{G}^{2}(-\Delta w) d x \leq \int w d \mu_{G}
$$

since $-\Delta w \geq 0$ and $0 \leq \phi_{G} \leq 1$. But since $\mu_{G}(E) \leq C(e)$ for all Borel sets $e$, it follows that

$$
\int\left|\nabla \phi_{G}\right|^{2} w d x \leq \int_{G} w d C
$$

And finally since $\phi_{G}=1$ on $G$ (and not just $C$-a.e.!), we get

$$
C^{w}(K) \leq C^{w}(G) \leq \int_{G} w d C .
$$

The final conclusion now follows by the capacitibility results of Choquet referred to earlier.

To prove part (b), we use (4). Let $\phi \in C_{0}^{\infty}\left(\mathbb{R}^{n}\right)^{+}$such that $\phi \geq 1$ on $K$, then

$$
\int_{K} w d C \leq \int\left[w^{1 / 2} \phi\right]^{2} d C \leq Q \int|\nabla \phi|^{2} w d x+Q^{\prime} \int \phi^{2} \frac{|\nabla w|^{2}}{w} d x
$$

From $\left[\mathbf{A}_{5}\right]$ it follows that there is a constant $Q$ such that

$$
\int \phi^{2} \frac{|\nabla w|^{2}}{w} d x \leq Q \int|\nabla \phi|^{2} w d x
$$

if and only if

$$
\sup _{K} C^{w}(K)^{-1} \int_{K} \frac{|\nabla w|^{2}}{w} d x<\infty
$$

Thus with (15),

$$
\int_{K} w d C \leq Q^{\prime \prime} \int|\nabla \phi|^{2} w d x
$$

and (10) follows. The only trouble with (15) is that it is hard to check, although it can be shown that if $w \in L^{p}$ for sufficiently large $p$, then (15) holds. We are, however, concerned with a different set of hypotheses. In fact, if we apply Theorem 7.3 from $\left[\mathbf{A}_{5}\right]$ to $w=I_{2} * v$, we see that to get (14) it is sufficient to show that for some $s>1$

$$
\left[f_{B}\left(\frac{|\nabla w|^{2}}{w}\right)^{s} d x\right]^{1 / s} \leq Q \cdot|B|^{-2 / n} f_{B} w d x
$$

for all balls $B \subset \mathbf{R}^{n}$. But from $[\mathbf{H}]$, we can write

$$
|\nabla w|^{2} / w \leq Q\left(I_{1} * v\right)^{2} / I_{2} * v \leq Q^{\prime} \cdot M_{0} v
$$

for some constants $Q$ and $Q^{\prime}$. Thus since we always have

$$
|B|^{-2 / n} f_{B} I_{2} * v d x \geq Q \cdot f_{B} v
$$


it then suffices to prove the inequality

$$
\left[f_{B}\left(M_{0} v\right)^{s} d x\right]^{1 / s} \leq Q \cdot f_{B} v
$$

for some constant $Q$. This inequality follows from two facts, both of which are a consequence of our assumption $v \in A_{\infty}$. They are

(i) $v$ satisfies the reverse Hölder inequality

$$
\left(f_{B} v^{s} d x\right)^{1 / s} \leq Q \cdot f_{B} v
$$

for all balls $B, Q$ a constant, and some $s>1$.

(ii) $v^{s}$ has the doubling property, i.e.

$$
v^{s}(B(x, \rho t)) \leq Q \rho^{n \mu} v^{s}(B(x, t))
$$

for all $\rho \geq 1 ; 0<\mu \leq 1$, and $v^{s}(B)$ means that $v^{s}$ is integrated (with Lebesgue measure) over $B$.

For (i) and (ii) as a consequence of our assumption, see [CF]. So for (17) we split up $v=v^{\prime}+v^{\prime \prime}$ where $v^{\prime}$ is just $v$ on $2 B$ and zero otherwise. Then

$$
\int_{B}\left(M_{0} v^{\prime}\right)^{s} d y \leq Q \int_{2 B} v^{s} d y
$$

and for $x \in B=B\left(x_{0}, r\right)$

$$
\frac{1}{t^{n}} \int_{B(x, t)} v^{\prime \prime} d y \leq Q\left[\left(\frac{r}{t}\right)^{n(1-\mu)}\right]^{1 / s} \cdot\left(f_{B(x, r)} v^{s} d y\right)^{1 / s}
$$

for $t \geq r$. Thus (17) holds.

\section{REFERENCES}

[A $\left.\mathbf{A}_{1}\right]$ D. R. Adams, Maximal operators and capacity, Proc. Amer. Math. Soc. 34 (1972), $152-156$.

$\left[\mathbf{A}_{2}\right]-$ Sets and functions of finite $L^{p}$-capacity, Indiana Univ. Math. J. $27(1978), 611-627$.

$\left[\mathbf{A}_{3}\right]-$ A note on differentiation with respect to $L^{p}$-capacity, preprint, Univ. of Kentucky, 1978, $8 \mathrm{pp}$.

$\left[\mathbf{A}_{4}\right] \longrightarrow$, Lectures on $L^{p}$-potential theory, Umeå Univ. Reports, No. 2, 1981.

$\left[\mathbf{A}_{5}\right]$, Weighted nonlinear potential theory, Trans. Amer. Math. Soc. 297 (1986), 73-94.

[An] B. Anger, Representation of capacities, Math. Ann. 229 (1977), 245-258.

[BZ] T. Bagby and W. Ziemer, Pointwise differentiability and absolute continuity, Trans. Amer. Math. Soc. 191 (1974), 129-148.

[C $\left.\mathbf{C}_{1}\right]$ G. Choquet, Theory of capacities, Ann. Inst. Fourier 5 (1953), 131-295.

$\left[\mathbf{C}_{2}\right]$, Forme abstraite du theoreme de capacitabilitie, Ann. Inst. Fourier 9 (1959), 83-89.

[CF] R. Coifman and C. Fefferman, Weighted norm inequalities for maximal functions and singular integrals, Studia Math. 60 (1974), 241-250.

[EP] D. E. Edmunds and L. A. Peletier, A Harnack inequality for weak solutions of degenerate quasilinear elliptic equations, J. London Math. Soc. 5 (1972), 21-31.

[FKS] E. Fabes, C. Kenig, and R. Serapioni, The local regularity of solutions of degenerate elliptic equations, Comm. Partial Differential Equations 7 (1982), 77-116.

[FJK] E. Fabes, D. Jerison, and C. Kenig, The Wiener test for degenerate elliptic equations, Ann. Inst. Fourier 32 (1982), 151-182.

[Ha] K. Hansson, Imbedding theorems of Sobolev type in potential theory, Math. Scand. 45 (1979), 77-102.

[H] L. I. Hedberg, On certain convolution inequalities, Proc. Amer. Math. Soc. 36 (1972), 505-510. 
[LSW] W. Littman, G. Stampacchia, and H. Weinberger, Regular points for elliptic equations with discontinuous coefficients, Ann. Scuola Norm. Sup. Pisa 17 (1963), 45-79.

[M] N. Meyers, A theory of capacities for potentials of functions in Lebesgue classes, Math. Scand. 26 (1970), 255-292.

[MS] M. K. Murthy and G. Stampacchia, Boundary value problems for some degenerate elliptic operators, Ann. Mat. Pura Appl. 80 (1968), 1-122.

[S] G. Stampacchia, Le probleme de Dirichlet pour les equations elliptique du second order á coefficients discontinuous, Ann. Inst. Fourier (Grenoble) 15 (1965), 189-258.

[St] E. Stein, Maximal functions: Spherical means, Proc. Nat. Acad. Sci. U.S.A. 73 (1976), 21742175.

Department of. Mathematics, University of Kentucky, Lexington, KenTUCKY 40506-0027 